\title{
Passivity Test of Immittance Descriptor Systems Based on Generalized Hamiltonian Methods
}

\author{
Zheng Zhang, Student Member, IEEE, and Ngai Wong, Member, IEEE
}

\begin{abstract}
A generalized Hamiltonian method (GHM) and its half-size variant (HGHM) are proposed to characterize the spectral behaviors of descriptor systems (DSs). With the preprocess improper part test, GHM and HGHM can be applied to test the passivity of immittance (impedance or admittance) DSs without system decomposition, system index assumption, or minimal realization requirement, which are the major bottlenecks of existing algebraic DS passivity tests. The proposed method allows exact detection of nonpassive frequency intervals, which is not possible with frequency-sweeping techniques. Numerical results confirm the effectiveness of the proposed methods.
\end{abstract}

Index Terms-Descriptor system (DS), generalized Hamiltonian method (GHM), half-size GHM (HGHM), passivity.

\section{INTRODUCTION}

$\mathbf{T}$ HIS brief is motivated by the demand of passive modeling of on-chip components and electrical circuits in VLSI simulations [1], [2]. Passivity can be interpreted as the inability of a system to internally generate energy, which is of great importance for stable global simulations. However, nonpassive models may be generated from some stability-preserving algorithms (e.g., [3]) or even some theoretically passivitypreserving techniques (e.g., [1]) on finite-precision machines. As a remedy, passivity enforcement techniques [2] can eliminate or mitigate passivity violations. These enforcements need to locate the possible nonpassive regions via a passivity test in advance. For regular (or nonsingular) systems, numerous passivity assessments have been proposed. The reader is referred to [2] and [4], and the references therein.

As a superset of regular state-space systems, descriptor systems (DSs) [6], [7] are widely used in VLSI simulations [2], [8]. Nevertheless, DS passivity tests are much less developed compared with their regular system counterparts. The $O\left(n^{6}\right)$ computation renders the extended linear matrix inequation tests [8] impractical for general DSs. Chu and Tan [9] presented a cheaper method based on the generalized Schur decomposition, but it posed strict restrictions on system observability and controllability. Some assess positive realness via generalized algebraic Riccati equations (GAREs) [10], but the admissible requirement is also very strong for practical physical models.

Manuscript received July 20, 2009; revised September 15, 2009. First published January 8, 2010; current version published January 15, 2010. This work was supported in part by the Hong Kong Research Grants Council under GRF projects HKU 717407E and 718509E, and in part by the University Research Committee of The University of Hong Kong. This paper was recommended by Associate Editor J. Lu.

The authors are with the Department of Electrical and Electronic Engineering, The University of Hong Kong, Hong Kong (e-mail: zzhang@eee.hku.hk; nwong@eee.hku.hk).

Color versions of one or more of the figures in this paper are available online at http://ieeexplore.ieee.org.

Digital Object Identifier 10.1109/TCSII.2009.2037263
Furthermore, none of these methods can locate the nonpassive frequency regions. Some decompose-and-test flows [11], [12] require the DSs to be minimal, and the system decomposition and transformation may induce large numerical errors. The eigenvalue-based DS passivity test [13] is only applicable to scalar functions. Frequency-sweeping methods [14] detect nonpassive regions at a set of frequency points, but they may miss nonpassive frequency intervals.

We propose, for the first time, a flexible passivity test for general DSs based on generalized Hamiltonian methods (GHMs). The main contributions of this brief include the following: 1) GHM and the half-size GHM (HGHM) to characterize the eigenvalues of DS spectral functions; 2) a complete DS passivity test based on the improper part test (ImPT), GHM, and HGHM to easily test the improper and proper parts without system decomposition; and 3) the observation that the GHMand HGHM-based passivity tests are the supersets of the traditional Hamiltonian method and its half-size [4] counterpart, respectively, as well as the connection of GHM with GAREs [10]. A preliminary version of this brief, which does not contain HGHM and related results, was presented in [15].

\section{Preliminaries of LTI System Passivity}

For an immittance linear time-invariant (LTI) system, the (strict) passivity is equivalent to its square transfer matrix $H(s)$ being (strictly) positive real [9].

1) $H(s)$ has no poles in $\boldsymbol{R e}[s]>0$.

2) $\overline{H(s)}=H(\bar{s})$, where $\bar{o}$ stands for the conjugate of $o$.

3) The spectral function $G(j \omega)=\left(H(j \omega)+H^{*}(j \omega)\right) / 2 \geq$

0 for all $\omega \in \mathbb{R}$ (> for strict positive realness), where ${ }^{*}$ means the conjugate transpose operation.

For a regular state-space system $H(s)=C(s I-A)^{-1} B+$ $D$, its passivity can be tested by the Hamiltonian matrix [5]

$$
M=\left[\begin{array}{cc}
\hat{A} & -\hat{R} \\
\hat{P} & -\hat{A}^{T}
\end{array}\right]
$$

of which any purely imaginary eigenvalue defines a boundary frequency of passivity violations. In (1), $\hat{A}=A-$ $B\left(D+D^{T}\right)^{-1} C, \hat{R}=B\left(D+D^{T}\right)^{-1} B^{T}$, and $\hat{P}=C^{T}(D+$ $\left.D^{T}\right)^{-1} C$.

In circuit or system simulations, we usually use the LTI DS

$$
E \dot{x}=A x+B u \quad y=C x+D u
$$

where $x \in \mathbb{R}^{n}$ denotes the state variables, $E, A \in \mathbb{R}^{n \times n}, B$, $C^{T} \in \mathbb{R}^{n \times m}, D \in \mathbb{R}^{m \times m}$, and $\operatorname{rank}(E) \leq n$ ("=" corresponds to regular cases). The transfer matrix of (2) is

$$
H(s)=C(s E-A)^{-1} B+D .
$$


Here, $(A, E)$ is assumed to be regular, i.e., $\operatorname{det}(s E-A)$ is not identically zero. There exists a Weierstrass form [9]

$$
(A, E)=W\left(\left[\begin{array}{cc}
F & 0 \\
0 & I_{n-q}
\end{array}\right],\left[\begin{array}{cc}
I_{q} & 0 \\
0 & N
\end{array}\right]\right) T
$$

where $W$ and $T$ are nonsingular, $I_{q}$ denotes an identity matrix of dimension $q$, and $F$ and $N$ (an index- $\mu$ nilpotent matrix, i.e., $N^{\mu}=0$ and $N^{\mu-1} \neq 0$ ) correspond to the finite and infinite generalized eigenvalues of $(A, E)$, respectively. The Weierstrass form shows that

$$
H(s)=\underbrace{C_{p}\left(s I_{q}-F\right)^{-1} B_{p}+M_{0}}_{H_{p}(s)}+\underbrace{\sum_{k=1}^{\mu-1} s^{k} M_{k}}_{H_{\mathrm{imp}}(s)}
$$

where $\left[\begin{array}{ll}C_{p} & C_{\infty}\end{array}\right]=C T^{-1},\left[\begin{array}{c}B_{p} \\ B_{\infty}\end{array}\right]=W^{-1} B, M_{0}=D-$ $C_{\infty} B_{\infty}$, and $M_{k}=-C_{\infty} N^{k} B_{\infty}(k=1, \ldots, \mu-1) . H_{p}(s)$ and $H_{\mathrm{imp}}(s)$ are the proper and improper parts, respectively.

The immittance DS in (5) is passive if and only if [9] $H_{p}(s)$ is passive; $M_{1} \geq 0$ and $M_{k}=0$ for any $k \geq 2$.

\section{GHM AND HGHM THEORIES FOR DSs}

\section{A. GHM for General DSs}

Theorem 1: Assume that $\lambda$ is not an eigenvalue of $(D+$ $\left.D^{T}\right) / 2$ for the DS in (2) (i.e., any finite $s$ satisfying $\operatorname{det}(A-$ $s E)=0$ is located on the left half-plane), then $\lambda$ is an eigenvalue of $G(j \omega)$ if and only if $j \omega$ is a generalized eigenvalue of the matrix pencil $(J, K)$, which is defined as

$$
(J, K)=\left(\left[\begin{array}{cc}
A+B Q^{-1} C & B Q^{-1} B^{T} \\
-C^{T} Q^{-1} C & -A^{T}-C^{T} Q^{-1} B^{T}
\end{array}\right],\left[\begin{array}{cc}
E & 0 \\
0 & E^{T}
\end{array}\right]\right)
$$

where $Q=\left(2 \lambda I-D-D^{T}\right)$.

Proof: Assume that $\lambda$ is an eigenvalue of $G(j \omega)$. Since $H^{*}(j \omega)=H^{T}(-j \omega)$, we have $x \neq 0$ such that

$$
2 G(j \omega) x=\left\{\left[\begin{array}{ll}
C & B^{T}
\end{array}\right] \Omega_{\omega}^{-1}\left[\begin{array}{c}
B \\
-C^{T}
\end{array}\right]+D+D^{T}\right\} x=2 \lambda x .
$$

Here, $\Omega_{\omega}=\left[\begin{array}{ll}j \omega E-A & \\ & j \omega E^{T}+A^{T}\end{array}\right]$. We rewrite (7) as

$$
Q^{-1}\left[\begin{array}{ll}
C & B^{T}
\end{array}\right] z=x
$$

with $z=\Omega_{\omega}^{-1}\left[\begin{array}{c}B \\ -C^{T}\end{array}\right] x \neq 0$. Equation (8) implies that

$$
\Omega_{\omega}^{-1}\left[\begin{array}{c}
B \\
-C^{T}
\end{array}\right] Q^{-1}\left[\begin{array}{ll}
C & B^{T}
\end{array}\right] z=z
$$

which is equivalent to

$$
J z=j \omega K z .
$$

Conversely, denoting $w:=Q^{-1}\left[\begin{array}{ll}C & B^{T}\end{array}\right] z[w \neq 0$, observed in (9)] and premultiplying both sides of (9) by $Q^{-1}\left[\begin{array}{ll}C & B^{T}\end{array}\right]$, we reach

$$
Q^{-1}\left[\begin{array}{ll}
C & B^{T}
\end{array}\right] \Omega_{\omega}^{-1}\left[\begin{array}{c}
B \\
-C^{T}
\end{array}\right] w=w
$$

which is equivalent to (7); thus, $\lambda$ is an eigenvalue of $G(j \omega)$.

\section{B. HGHM for Symmetric DSs}

Theorem 2: For symmetric DSs, if $\lambda$ is not an eigenvalue of $D,(J, K)$, which is defined in (6), reduces to

$$
\left(J_{h}, K_{h}\right)=\left(A+B(\lambda I-D)^{-1} C, E A^{-1} E\right)
$$

and the generalized eigenvalue $j \omega$ is replaced by $\beta=\omega^{2}$.

Proof: For symmetric DSs, $H^{*}(j \omega)=H(-j \omega)=$ $-C(j \omega E+A)^{-1} B+D$, and $(J, K)$ can be written as

$$
(J, K)=\left(\left[\begin{array}{cc}
S & T \\
-T & -S
\end{array}\right],\left[\begin{array}{cc}
E & 0 \\
0 & E
\end{array}\right]\right)
$$

where $\quad S=A+B(2 \lambda I-2 D)^{-1} C, \quad$ and $\quad T=B(2 \lambda I-$ $2 D)^{-1} C$. We set $Z=\left[\begin{array}{cc}I & I \\ I & -I\end{array}\right]$, then $\left(J^{\prime}, K^{\prime}\right)=Z(J, K) Z^{T}$ has the same generalized eigenvalues with $(J, K)$, where

$$
\left(J^{\prime}, K^{\prime}\right)=\left(\left[\begin{array}{cc}
0 & 2(S-T) \\
2(S+T) & 0
\end{array}\right],\left[\begin{array}{cc}
2 E & \\
& 2 E
\end{array}\right]\right) .
$$

Assume that $\lambda$ is an eigenvalue of $G(j \omega)$, then $j \omega$ is also a generalized eigenvalue of $\left(J^{\prime}, K^{\prime}\right)$, and there exists

$$
\left[\begin{array}{cc}
-j \omega E & S-T \\
S+T & -j \omega E
\end{array}\right]\left[\begin{array}{l}
x_{1} \\
x_{2}
\end{array}\right]=0 \quad\left[\begin{array}{l}
x_{1} \\
x_{2}
\end{array}\right] \neq 0
$$

which can further be reduced to

$$
\left(J_{h}-\omega^{2} K_{h}\right) x_{1}=0, \quad x_{1} \neq 0 .
$$

Therefore, $\beta=\omega^{2}$ is a generalized eigenvalue of $\left(J_{h}, K_{h}\right)$.

Conversely, setting $x_{2}=j \omega(S-T)^{-1} x_{1}$, we can arrive at (15) from (16) and then Theorem 1.

\section{DS PAssivity Test}

\section{A. Testing the Improper Part by ImPT}

Denoting the highest order of $H_{\mathrm{imp}}(s)$ by the integer $\zeta-$ $1(1 \leq \zeta \leq \mu)$, we propose ImPT to characterize the improper part of a DS. Given a set of positive real scalars $s_{i}(i=1,2, \ldots)$ with $s_{i+1}=\eta s_{i}(\eta>1)$, the norm of $H\left(s_{i}\right)$ is

$$
\left\|H\left(s_{i}\right)\right\|=s_{i}^{\zeta-1}\left\|M_{\zeta-1}+\frac{M_{\zeta-2}}{s_{i}}+\cdots+\frac{H_{p}\left(s_{i}\right)}{s_{i}^{\zeta-1}}\right\| .
$$

If $s_{i}$ is large enough, $s_{i}^{\zeta-1} M_{\zeta-1}$ dominates $H\left(s_{i}\right)$. In this case, we have $M_{\zeta-1}+\left(M_{\zeta-2} / s_{i}\right)+\cdots+\left(H_{p}\left(s_{i}\right) / s_{i}^{\zeta-1}\right) \approx M_{\zeta-1}$ and

$$
\frac{\left\|H\left(s_{i+1}\right)\right\|}{\left\|H\left(s_{i}\right)\right\|} \approx \eta^{\zeta-1} .
$$

Therefore, $\zeta$ can be computed by

$$
\zeta=\left[\log _{\eta}\left(\frac{\left\|H\left(s_{i+1}\right)\right\|}{\left\|H\left(s_{i}\right)\right\|}\right)\right]+1
$$

where $[o]$ represents rounding. In practice, we may start with randomly selected numbers (e.g., $s_{1}=10^{5}$ ) and then replace $s_{i}$ with $s_{i+1}$ until $\mid\left[\log _{\eta}\left(\left\|H\left(s_{i+1}\right)\right\| /\left\|H\left(s_{i}\right)\right\|\right)\right]-$ $\log _{\eta}\left(\left\|H\left(s_{i+1}\right)\right\| /\left\|H\left(s_{i}\right)\right\|\right) \mid<\delta$. Here, $\delta$ is a small positive constant used to control numerical errors. Since $s_{i}$ is exponentially increased, the iteration can converge very fast. If $\zeta \geq 3$, 


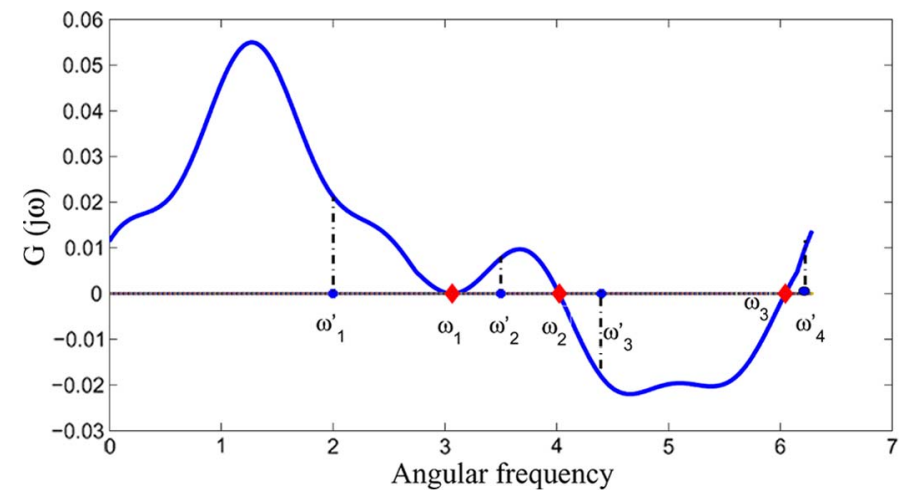

Fig. 1. Illustrative example for nonpassive region identification.

we have $M_{2} \neq 0$, and the DS is nonpassive. In the case $\zeta=2$, $H\left(s_{i}\right)=H_{p}\left(s_{i}\right)+H_{\text {imp }}\left(s_{i}\right) \approx s_{i} M_{1}+M_{0}$, then

$$
M_{1} \approx \frac{H\left(s_{i+1}\right)-H\left(s_{i}\right)}{s_{i+1}-s_{i}} .
$$

With a numerical error control, (20) can also be used to compute $M_{1}$ with high accuracy. In impulse-free DSs, the denominator in (19) might approach zero in the case $M_{0}=0$; thus, (19) may give erroneous results. In this case, we replace $H\left(s_{i}\right)$ with $H\left(s_{i}\right)+I_{m}$ to compute $\zeta$.

Hereafter, we assume that $H_{\mathrm{imp}}(s)$ has been checked by $\operatorname{ImPT}, \zeta \leq 2$, and $M_{1} \geq 0$. In this case, $H_{p}(j \omega)+H_{p}^{*}(j \omega)=$ $H(j \omega)+H^{*}(j \omega)$.

\section{B. Testing the Proper Part by GHM and HGHM}

Setting $\lambda=0$, we have $(J, K)=\left(J_{0}, K_{0}\right)$ with

$$
J_{0}=M=\left[\begin{array}{ll}
\hat{A} & -\hat{R} \\
\hat{P} & -\hat{A}
\end{array}\right] \quad K_{0}=K .
$$

Here, $M$ is the Hamiltonian matrix defined in (1). For HGHM, setting $\lambda=0$ gives a half-size matrix pencil

$$
\left(J_{h 0}, K_{h 0}\right)=\left(A-B D^{-1} C, E A^{-1} E\right) .
$$

Any purely imaginary (or positive real) generalized eigenvalue $j \omega$ (or $\beta=\omega^{2}$ ) of $\left(J_{0}, K_{0}\right)$ (or $\left(J_{h 0}, K_{h 0}\right)$ for symmetric DSs) defines a crossover angular frequency $\omega$. Assume that $\Theta=\left\{\omega_{1}, \ldots, \omega_{p}\right\}$, where $\omega_{i}(i=1, \ldots, p)$ denotes the $p$ crossover points obtained from GHM or HGHM; then the passive and nonpassive regions of $H(j \omega)$ can be identified as follows.

1) If $\Theta$ is empty, test $G\left(j \omega_{0}\right)$ at a randomly selected sampling point $\omega_{0}$. The system is strictly passive if $G\left(j \omega_{0}\right)>$ 0 ; otherwise, it is nonpassive at any frequency point.

2) If $\Theta$ is not empty, test $G\left(j \omega_{k}^{\prime}\right)$ at $\omega_{k}^{\prime} \in \ell_{k} \quad(k=$ $1,2, \ldots, p+1)$, where $\ell_{1}=\left(0, \omega_{1}\right), \ell_{i}=\left(\omega_{i-1}, \omega_{i}\right)$ for $i=2, \ldots, p$, and $\ell_{p+1}=\left(\omega_{p}, \infty\right)$. If $G\left(j \omega_{k}^{\prime}\right)>0$, then the DS is passive in the interval $\ell_{k}$; otherwise, it is nonpassive in $\ell_{k}$.

An illustrative example is shown in Fig. 1. For this DS, GHM and HGHM produce three crossover points. We randomly select one sampling point in each interval. Since $G\left(j \omega_{3}^{\prime}\right)<0$ and
$G\left(j \omega_{k}^{\prime}\right)>0$ for $k=1,2,4$, the DS is nonpassive in $\left(\omega_{2}, \omega_{3}\right)$ but passive in other frequency bands.

\section{Equivalent Model Conversion}

At first glance, the GHM/HGHM test requires $D+D^{T}$ to be nonsingular, which is not always satisfied in practical DSs. Assume that $\alpha \in \mathbb{R}$ is not an eigenvalue of $D$, then $D_{\alpha}=\alpha I-D$ is nonsingular. A new $\mathrm{DS} H^{\prime}(s)$ realized by $\left(E^{\prime}, A^{\prime}, B^{\prime}, C^{\prime}, D^{\prime}\right)$ can be constructed as

$$
\begin{array}{ll}
E^{\prime}=\left[\begin{array}{ll}
E & \\
& 0
\end{array}\right] & A^{\prime}=\left[\begin{array}{ll}
A & \\
& D_{\alpha}^{-1}
\end{array}\right] \\
B^{\prime}=\left[\begin{array}{c}
B \\
I
\end{array}\right] & C^{\prime}=\left[\begin{array}{ll}
C & I
\end{array}\right] \quad D^{\prime}=\alpha I .
\end{array}
$$

We note $H^{\prime}(s)=H(s)$, but $D^{\prime}+D^{\prime T}$ and $D^{\prime}$ are nonsingular. Therefore, the proper part of $H(s)$ can be assessed by testing the passivity of $H^{\prime}(s)$ via GHM or HGHM. The main computation in GHM and HGHM tests is the $O\left(n^{3}\right)$ generalized eigenvalue solution. The HGHM-based test should be $8 \times$ faster than the GHM-based method due to its half-size nature.

\section{Connection to the Traditional Hamiltonian Method and the Half-Size Singularity Test}

For standard state-space models $(E=I)$, the generalized eigenvalue solution of $(J, K)$ reduces to the eigenvalue solution of $J$ defined in (6). To check passivity, we set $\lambda=0$ and get $J_{0}=M$ [defined in (1)]. For symmetric regular systems, the generalized eigenvalue solution of $\left(J_{h 0}, K_{h 0}\right)$ in (22) can be replaced by the eigenvalue solution of

$$
M_{h}=A\left(A-B D^{-1} C\right)
$$

which is the half-size singularity test first proposed in [5].

Therefore, the Hamiltonian method and its half-size variant are special cases of GHM and HGHM, respectively. All of them detect passivity violation regions by finding boundary frequencies, but GHM and HGHM can deal with DSs and regular systems without restrictions on $D$. The complete test flow is illustrated in Fig. 2.

\section{E. Strict Positive Realness of Impulse-Free DSs}

The GARE [10] is widely used to characterize the positive realness of DSs. If $(A, E)$ is regular, impulse free, and $D+$ $D^{T}>0$, the following statements are equivalent.

1) $H(s)$ is strictly positive real.

2) The GARE

$$
\hat{A}^{T} X+X^{T} \hat{A}+X^{T} \hat{R} X+\hat{P}=0 \quad E^{T} X=X^{T} E \geq 0
$$

has a solution $X$ such that $(\hat{A}+\hat{R} X, E)$ is stable.

$3)$ The matrix pencil $\left(J_{0}, K_{0}\right)$ has no imaginary generalized eigenvalues and $M_{0}>0\left[M_{0}\right.$ is defined in (5)].

Proof: The equivalence of statements 1) and 2) has been proved in [11]. Statement 3) $\Rightarrow$ statement 1) is obvious. From statement 2), we get

$$
\begin{aligned}
& \operatorname{det}\left(J_{0}-s K_{0}\right) \\
& \quad=\operatorname{det}\left(\left[\begin{array}{cc}
I & \\
-X^{T} & I
\end{array}\right]\left(J_{0}-s K_{0}\right)\left[\begin{array}{cc}
I & \\
X & I
\end{array}\right]\right) \\
& \quad=\operatorname{det}(s E-(\hat{A}+\hat{R} X)) \operatorname{det}(s E+(\hat{A}+\hat{R} X)) .
\end{aligned}
$$




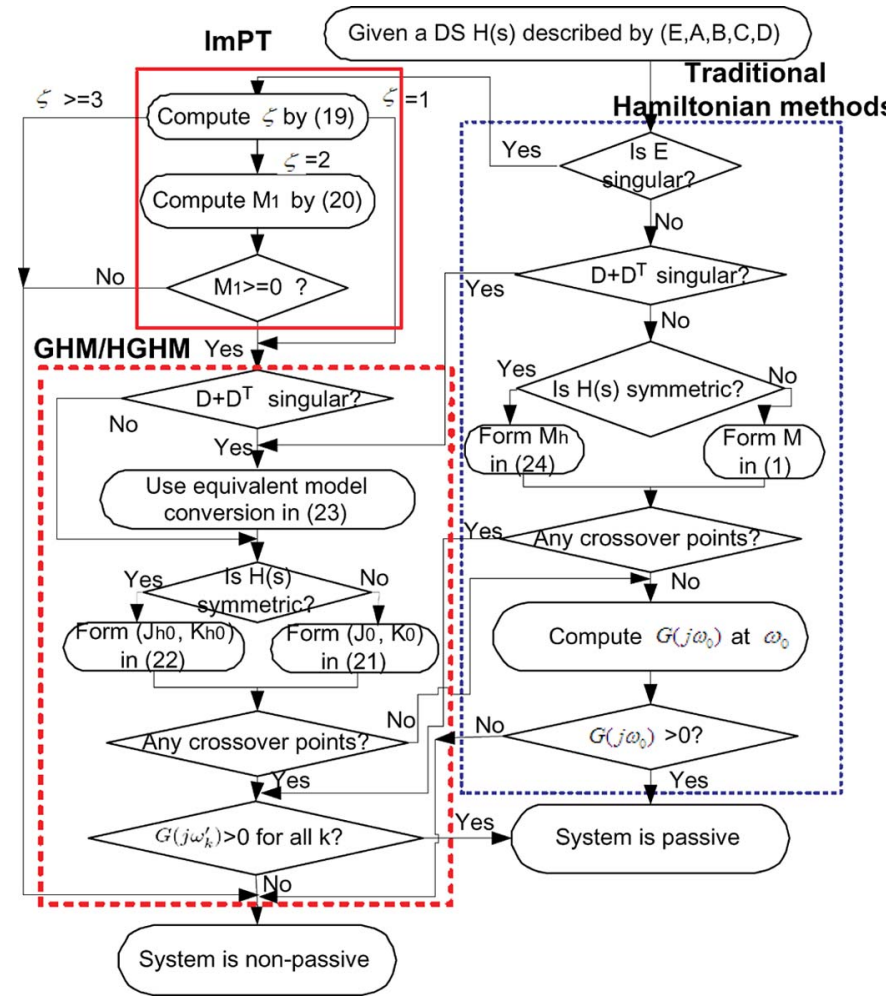

Fig. 2. Complete passivity test flow for DSs, including regular systems.
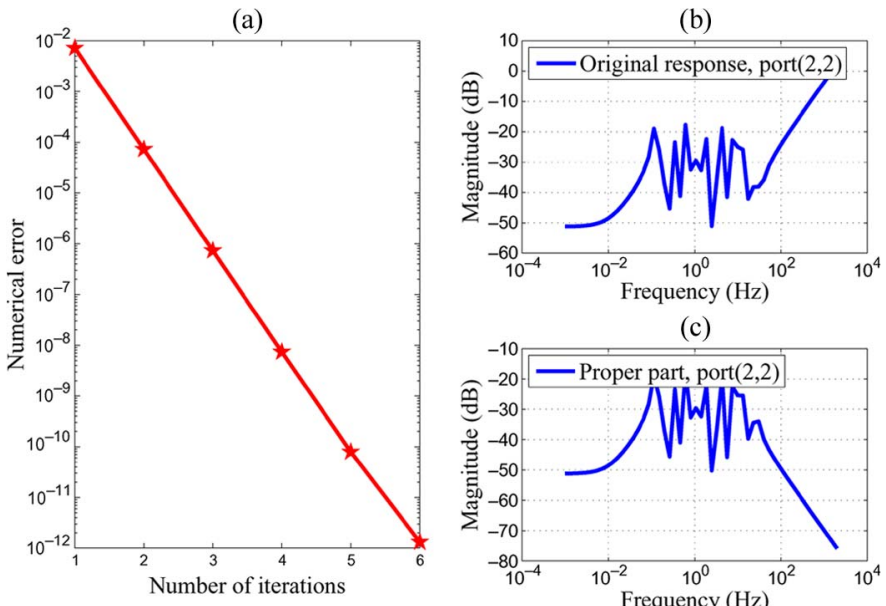

(c)

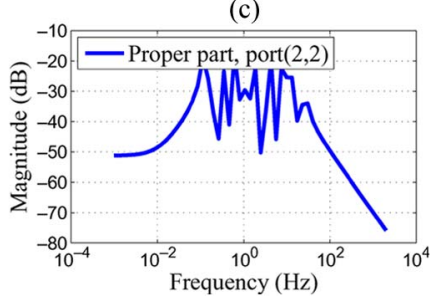

Fig. 3. ImPT for the modified nodal analysis (MNA) model [the iteration number refers to $i$ in (19)].

Since $(\hat{A}+\hat{R} X, E)$ is stable, $\hat{A}+\hat{R} X \pm j \omega E$ is nonsingular. Therefore, the matrix pencil $\left(J_{0}, K_{0}\right)$ has no imaginary eigenvalues. The equivalence of statements 1) and 2) also implies that $M_{0}>0$; therefore, statement 3) can be derived from statement 2), and the foregoing statements are equivalent.

We remark that the GARE requires $(E, A)$ to be impulse free, but GHM does not. Furthermore, GHM can locate the passive/nonpassive regions, whereas the GARE cannot.

\section{NumERICAL EXAMPLES}

This section presents some numerical examples to verify the proposed passivity test flow. All experiments are performed in MATLAB R2006a on a 2.66-GHz 2-GB RAM PC.

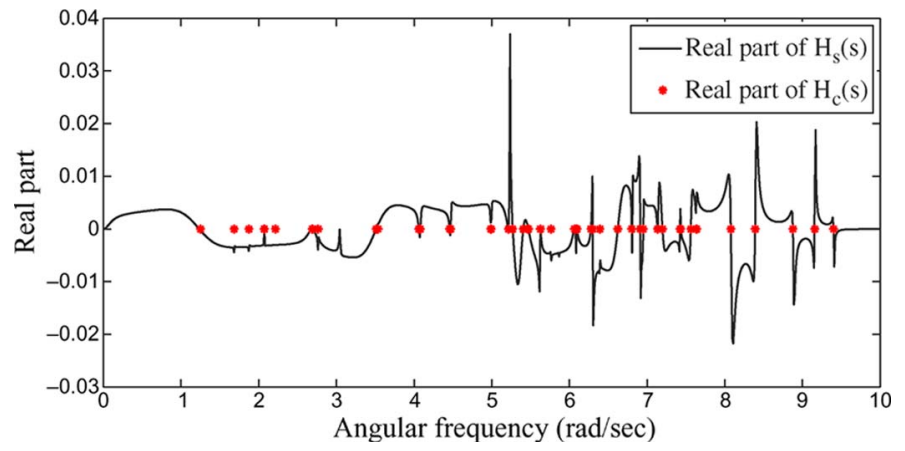

Fig. 4. GHM and frequency-sweeping results for the PEEC model.

TABLE I

GHM Test Results For the Reduced Model

\begin{tabular}{c|c}
\hline $\begin{array}{c}\text { Imaginary generalized } \\
\text { eigenvalues of }\left(J_{0}, K_{0}\right)\end{array}$ & $H_{r}(j \omega)$ \\
\hline \hline $3.65 \mathrm{e}-13 \pm j 3.3078$ & $0.0000+j 0.0031$ \\
\hline $5.00 \mathrm{e}-14 \pm j 1.2345$ & $0.0000-j 0.0060$ \\
\hline
\end{tabular}

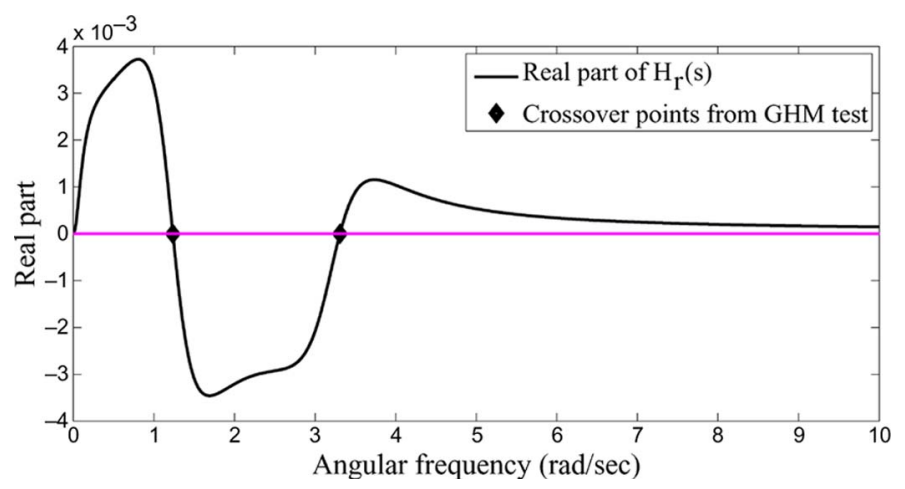

Fig. 5. Real part of $H_{r}(s)$.

1) MNA Example for ImPT: This order-10913 model describes a large $R L C$ network. Since the $R L C$ circuit is passive, $\zeta$ should be 1 or 2 , and $M_{1} \geq 0$. We first set $s_{1}=10^{3}$ and $\eta=10$ and plot the numerical error $\varepsilon_{i}$ for $i=1,2, \ldots, 6$ in Fig. 3(a), which shows that $\varepsilon_{i}$ decreases by two orders each iteration. Setting $s_{i}=10^{4}$ and $s_{i+1}=10^{5}$ in (19) yields $\zeta=2$ after $2.1 \mathrm{~s}$. The magnitude of port-2 to port-2 frequency response in Fig. 3(b) linearly increases in the high-frequency band, which also implies that $\zeta=2$. Via (20), we get a diagonal matrix with positive diagonal elements; thus, $M_{1}>0$. To verify the accuracy of $M_{1}$, we compute $\zeta$ of $H_{1}(s)=H(s)-s M_{1}$ by (19). We get $\zeta=1$ for $H_{1}(s)$, implying that $H_{1}(s)$ is impulse free, as expected. Meanwhile, the port- 2 to port- 2 response of $H_{1}(s)$ in Fig. 3(c) also shows that $H_{1}(s)$ has no impulsive part. This example shows that ImPT is efficient and accurate in practical implementations.

2) PEEC Example for GHM: The single-input-singleoutput order-51 reduced model is obtained by performing PRIMA [1] on a partial-element equivalent-circuit (PEEC) DS model of dimension 480 with $D=0$. ImPT shows that they are impulse free. The GHM test on the original model produces 59 crossover points. We compute the transfer functions at these 59 points [denoted by $H_{c}(s)$ ]. Fig. 4 shows that the real part of $H_{c}(s)$ is zero. By frequency sweeping, we get 29 boundary frequency points. The frequency-sweeping result 
TABLE II

GHM AND HGHM TEST RESUlTS FOR THE SAW MODEL

\begin{tabular}{c|c|c}
\hline $\begin{array}{c}\text { Imaginary results } \\
\text { of GHM }\end{array}$ & $\begin{array}{c}\text { Positive results } \\
\text { of HGHM }(\beta)\end{array}$ & $\sqrt{\beta}$ \\
\hline \hline$-1.4 \mathrm{e}-8 \pm j 21671.377$ & 469648579 & 21671.377 \\
\hline$-1.9 \mathrm{e}-8 \pm j 18029.84$ & 325075192.6 & 18209.84 \\
\hline $3.38 \mathrm{e}-8 \pm j 2645.316$ & 6997698.35 & 2645.316 \\
\hline
\end{tabular}

(a)
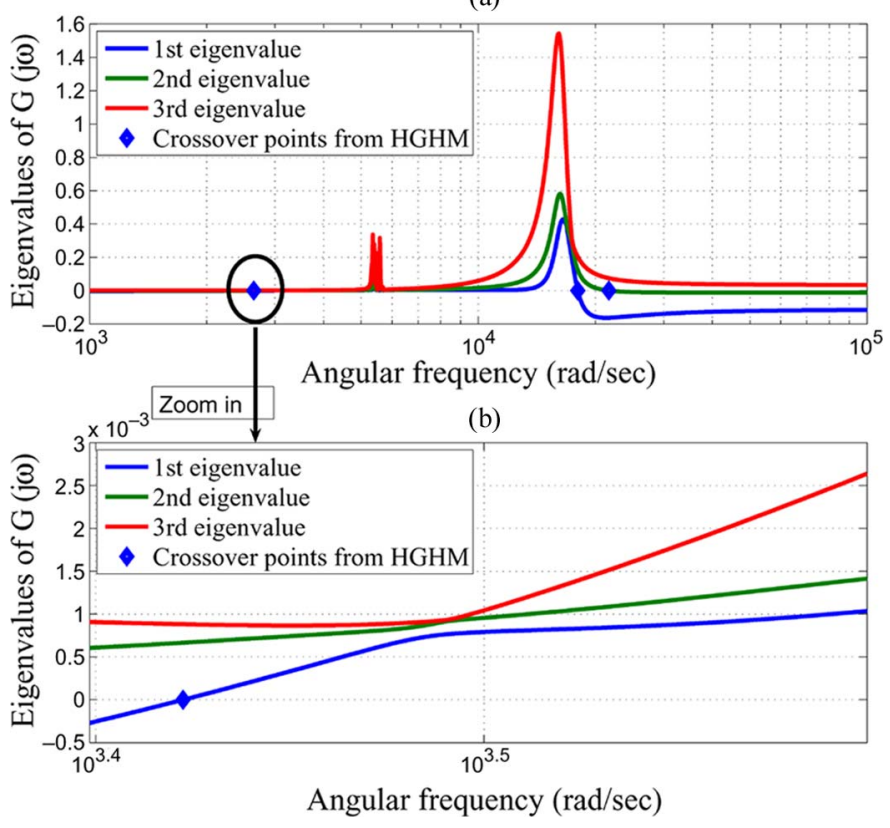

Fig. 6. HGHM test for the SAW model.

$H_{s}(s)$ is plotted in Fig. 4. All these 29 points are also detected by GHM. However, the other 30 crossover points are missed in the frequency-sweeping test. For the reduced model, GHM produces four purely imaginary results listed in Table I, which represent two crossover frequency points. We also plot the real part of the transfer function of the reduced model $\left(H_{r}(s)\right)$ in Fig. 5. The GHM results are accurately located at the crossover points of real $\left(H_{r}(s)\right)$ with the $x$-axis. GHM test results in Table I contain some numerical noise in the real parts, which is also observed in the traditional Hamiltonian method [4].

3) SAW Filter for HGHM: This order-126 admittance symmetric DS is from a three-terminal surface acoustic wave (SAW) filter. ImPT shows that this DS is impulse free. The HGHM test produces three positive real generalized eigenvalues, and the GHM test produces six imaginary generalized eigenvalues. Table II shows that the results from the GHM test have numerical noise in the real parts, but HGHM does not suffer from this problem. The three crossover points from HGHM are plotted in Fig. 6.

4) $C P U$ Time Comparison: We compare the CPU timing of GHM and HGHM with two decompose-and-test methods: skew-Hamiltonian/Hamiltonian (SHH) [11] and Weierstrass passivity tests [12]. For fairness, in decompose-and-test routines, the proper parts are tested by the Hamiltonian method. The CPU times of GHM, HGHM, SHH, and Weierstrass passivity tests are listed in Table III. It is shown that HGHM is about $8 \times$ faster than GHM. GHM is $(>2 \times)$ faster than SHH. The additional cost of SHH is mainly from system decompositions.
TABLE III

CPU Times of DifFERENT DS PASSIVIty TeSts (IN SECONDS)

\begin{tabular}{c|c|c|c|c}
\hline Model order & Weierstrass & SHH & GHM & HGHM \\
\hline \hline 150 & 1.1093 & 0.9375 & 0.3906 & 0.0625 \\
\hline 300 & 10.725 & 8.2500 & 3.3750 & 0.3906 \\
\hline 500 & 51.676 & 39.719 & 17.328 & 1.7788 \\
\hline 800 & 289.37 & 166.64 & 65.670 & 7.6406 \\
\hline
\end{tabular}

GHM, HGHM, and SHH are all faster than the Weierstrass test, which coincides with the observations in [11].

\section{CONCLUSION}

A new DS passivity test based on GHM/HGHM has been proposed for the first time. The most significant advantage of this method is its ability of accurately detecting the possible nonpassive regions, some of which may be missed with frequency-sweeping methods. With ImPT and equivalent model conversion, GHM and HGHM are applicable to general and symmetric DSs, respectively, without system decompositions. Experiments have demonstrated much higher accuracy of GHM than frequency sweeping, as well as faster computation than SHH and Weierstrass tests. In symmetric DSs, HGHM enjoys $8 \times$ speedup and higher numerical accuracy over GHM.

\section{REFERENCES}

[1] A. Odabasioglu, M. Celik, and L. Pileggi, "PRIMA: Passive and reducedorder interconnect macromodeling algorithm," IEEE Trans. Comput. Aided Design Integr. Circuits Syst., vol. 17, no. 8, pp. 645-654, Aug. 1998.

[2] S. Grivet-Talocia and A. Ubolli, "A comparative study of passivity enforcement schemes for linear lumped macromodels," IEEE Trans. Adv. Packag., vol. 31, no. 4, pp. 673-683, Nov. 2008.

[3] B. Gustavsen and A. Semlyen, "Rational approximation of frequency domain responses by vector fitting," IEEE Trans. Power Del., vol. 14, no. 3, pp. 1052-1061, Jul. 1999.

[4] A. Semlyen and B. Gustavsen, "A half-size singularity test matrix for fast and reliable passivity assessment of rational models," IEEE Trans. Power Del., vol. 24, no. 1, pp. 345-351, Jan. 2009.

[5] E. L. Yip and R. F. Sincovec, "Solvability, controllability and observability of continuous descriptor systems," IEEE Trans. Autom. Control, vol. AC-26, no. 3, pp. 702-707, Jun. 1981

[6] L. Dai, Singular Control Systems, vol. 118, Lecture Notes in Control and Information Sciences. Berlin, Germany: Springer-Verlag, 1989.

[7] H. Heeb and A. Ruehli, "Three-dimensional interconnect analysis using partial element equivalent circuits," IEEE Trans. Circuits Syst. I, Reg. Papers, vol. 39, no. 11, pp. 974-982, Nov. 1993.

[8] R. Freund, "An extension of positive real lemma to descriptor systems," Optim. Methods Softw., vol. 19, no. 1, pp. 69-87, Feb. 2004.

[9] D. Chu and R. C. E. Tan, "Algebraic characterizations for positive realness of descriptor systems," SIAM J. Matrix Anal. Appl., vol. 30, no. 1, pp. 197-222, Feb. 2008.

[10] L. Zhang, J. Lam, and S. Xu, "On positive realness of descriptor systems," IEEE Trans. Circuits Syst. I, Fundam. Theory Appl., vol. 49, no. 3, pp. 401-407, Mar. 2002.

[11] N. Wong and C. Chu, "A fast passivity test for stable descriptor systems via skew-Hamiltonian/Hamiltonian matrix pencil transformations," IEEE Trans. Circuits Syst. I, Reg. Papers, vol. 55, no. 2, pp. 635-643, Mar. 2008.

[12] P. Benner and D. Chu, "A new test for passivity of descriptor systems," Oberwolfach Report, vol. 1, no. 1, pp. 571-574, Nov. 2005.

[13] Z. Bai and R. Freund, "Eigenvalue-based characterization and test for positive realness of scalar transfer functions," IEEE Trans. Autom. Control, vol. 45 , no. 12 , pp. 2396-2402, Dec. 2000.

[14] Y. Liu and N. Wong, "Fast sweeping methods for checking passivity of descriptor systems," in Proc. IEEE Asia Pacific Conf. Circuits Syst., Dec. 2008 , pp. 566-569.

[15] Z. Zhang, C. U. Lei, and N. Wong, "GHM: A generalized Hamiltonian method for passivity test of impedance/admittance descriptor systems," in Proc. Int. Conf. Comput.-Aided Des., San Jose, CA, Nov. 2009, pp. 767-773. 